\title{
Interrogation Methods and Terror Networks
}

\author{
Mariagiovanna Baccara and Heski Bar-Isaac*
}

March 10, 2008

\begin{abstract}
We examine how the structure of terror networks varies with legal limits on interrogation and the ability of authorities to extract information from detainees. We assume that terrorist networks are designed to respond optimally to a trade-off caused by information exchange: Diffusing information widely leads to greater internal efficiency, but it leaves the organization more vulnerable to law enforcement. The extent of this vulnerability depends on the law enforcement authority's resources, strategy and interrogation methods. Recognizing that the structure of a terrorist network responds to the policies of law enforcement authorities allows us to begin to explore the most effective policies from the authorities' point of view.
\end{abstract}

\section{Introduction}

Apprehending terrorists and extracting information from them and, in particular, the different investigation and interrogation methods used are the topics of an ongoing debate which once again took center stage upon the start of the U.S. war on terror. ${ }^{1}$ In this article, we do not aim to examine the ethical and legal aspects of the debate, but, highlight the fact that the efficacy of different methods may vary to the extent that the structure of terror networks strategically adapts to different law enforcement policies.

The starting point for our work is the observation that the way terrorist networks are designed is the result of a trade-off: Diffusing information throughout the organization

${ }^{*}$ Department of Economics, Stern School of Business, New York University, 44 W Fourth Street, New York, NY 10012. Email addresses: mbaccara@stern.nyu.edu and heski@nyu.edu.

${ }^{1}$ A similar debate took place, for example, at the beginning of the $1900 \mathrm{~s}$, during the engagement of the U.S. against the Spanish colony in the Philippines. The media reported cruelties against Filipino prisoners to gather information on the counter-insurgency (see "The Water Cure" by P. Kramer, The New Yorker, $2 / 25 / 2008)$. 
allows it to operate more successfully, but it leaves the organization more vulnerable to law enforcement detection. In choosing their design, terror organizations act as strategic entities that respond optimally to the policies implemented by the authorities. Empirically, it certainly appears that protecting information is a crucial concern of terror networks since their survival depends on how effective they are in preventing information leakage. The available evidence suggests that terror network are characterized by a fairly decentralized "cell" structure; however, there are some agents that appear to hold more information than others (which we term an "informational hub"). ${ }^{2}$

In this article, we first aim to describe how authorities should expect the organization to respond strategically to alternative policies. In particular, we highlight the role and the consequences of investigation and interrogation methods on the information structure of the terror organization. Given our findings, we take the authorities' point of view and discuss the problem of choosing the most effective policy to fight terror networks.

It is worth highlighting from the outset, what we mean by the "network" and the costs and benefits of "informational links." ${ }^{3}$ We focus on a directed network and, specifically, if Bob is linked to Ann, this should be understood as Ann having some information about Bob. This information could, for example, allow Ann to ensure that Bob acts in the interests of the group. Examples of this kind of information include identity, whereabouts, or incriminating evidence about a person. ${ }^{4}$ We view the network of who holds such information as independent of the other structures that coexist within the organization. For example, planning, decision-making, coordinating activity and communication can take place under code-names. ${ }^{5}$ In that case, coordinating actions can be quite independent of who within the organization knows the real name or whereabouts of other members of the

\footnotetext{
${ }^{2}$ See, for instance, Arquilla and Ronfeldt (2002).

${ }^{3}$ In particular, within any organization (and terrorist organizations are no exception) many networks coexist simultaneously and often interact: ranging from the network of decision-making authority, to communication networks, "productive networks" who act and work together, and more informal networks based on social interactions. Moreover, networks can vary in whether they are directed (for example, an authority network where Ann may be able to veto Bob's decisions) or undirected (for example. if Ann is Bob's cousin, then it's a safe bet that Bob is Ann's cousin).

${ }^{4}$ Though these examples are drawn from gangs and crime rather than from terrorist structures, Thompson (2005), for example, describes that in his role as a journalist reporting on organized crime, he had to divulge his address and that of his close family members. Charlie (2002) describes how committing a murder in front of peers is often an initiation ritual used by U.S. gang members.

${ }^{5}$ Indeed, this was exactly what was done, for example, by the Italian resistance in WWII (see, for instance, Oliva (1976)) . Moreover, the investigations on Al-Qaeda suggest that the same Al-Qaeda member often uses different names depending on whom he is interacting with, as described, for instance, in the account of the investigation following Daniel Pearl's murder in Pearl (2003).
} 
organization. However, the diffusion of this kind of information clearly has implications both for the extent of trust (and the efficient functioning of the organization) and the organization's vulnerability with respect to its enemies.

In the model, we simply assume a fixed benefit of linking one member of the organization to another. This benefit can be viewed as arising from the enhanced ability of the organization to discipline such a member, leading to better outcomes from the group's perspective. ${ }^{6}$ Here, we devote more attention to fleshing out the costs of the network, and how they respond to law enforcement policies. In particular, we suppose that individuals within the organization are directly vulnerable according to the authorities' investigation policies. Further, if caught, then a member of the network will jeopardize other members of the network, depending on the extent to which he has information about them (that is, depending on whether they are linked to him), and depending on the law enforcement's ability to extract such information through interrogations.

Since we view the structure of the network as designed in response to the costs (of vulnerability to the authorities) and benefits of information links, we can characterize the network that arises in any set of circumstances, and show how different law enforcement capabilities, resources or policies affect the structure of the terrorist network. In particular, we show that both centralized and decentralized networks can arise as optimal responses to investigation policies. On the one hand, given a certain investigation budget, the optimal network tends to be more decentralized when the authorities invest similar resources to seek each agent. On the other hand, when the budget is allocated asymmetrically, the optimal organization is a mixed structure in which one cell acts as "information hub" and holds information about a certain number of members of the organization. However, there are other members of the organization that remain independent from the hub and are organized in binary cells. We also find that sometimes, as the interrogation techniques become harsher, the terror network responds by increasing the number of cells in the mixed structure. Therefore, our results suggest that there are circumstances in which harsher interrogation methods do not change the number of links in the organization (and, so, do not affect its operation), but just have the effect of increasing the degree of decentralization in the way information is shared in the network.

After we characterize the effect of different policies on network structure, we can turn to the assessment of the authorities' policies and, therefore, to the characterization of the

\footnotetext{
${ }^{6}$ Such a benefit is derived endogenously in Baccara and Bar-Isaac (2008).
} 
optimal policies. Our results suggest that the three instruments available to the authorities (investigation methods, interrogation methods and severity of punishments) are not independent but are strategically interrelated. If interrogation methods and severity of punishments are bounded by legal limits, the investigation methods should be tailored to the legal environment. We show the circumstances under which a symmetric investigation strategy (i.e., allocating the same budget to detect all potential members of the organization) is optimal, and we also look at how optimal investigation strategies vary with changes in the legal environment within which the authorities act. In particular, we find that as legal limits broaden, the cost of a link in a binary cell increases. This makes it more likely that a symmetric allocation of the investigation budget can prevent any links from arising. However, as legal boundaries become narrower, it is more likely that a symmetric budget allocation leads to a fully efficient allocation. In this case, we show that the authorities can do better by pursuing an asymmetrical investigation strategy.

\subsection{Related Literature}

This article builds on Baccara and Bar-Isaac (2008), which introduced a model that is more explicit on how information links lead to trust within the organization, and that allows for the probability of detection to vary with the extent of a terrorist's cooperation with the organization. Baccara and Bar-Isaac (2008) also provides some additional discussion of assumptions, and considers a number of extensions. Here, we simplify the model in a number

of respects - in particular, in presenting a reduced form benefit for an informational linkand extend the model to consider variety in interrogation methods. To our knowledge, this work is the first to address the optimal information structure in organizations subject to an external threat.

To our knowledge, this is the first article that addresses a terror network's strategic response to changes in the investigation and interrogation methods. However, several papers have some elements that are related to our work. Farley (2003, 2006) considers the robustness of a terrorist cell. In that work, robustness is with regard to maintaining a chain of command in a hierarchy. Garoupa (2007) looks at the organizational problem of an illegal activity and at the trade-off between enhancing internal productivity and leaving members of the organization more exposed to detection. He takes a different approach, focusing on the optimal size of the criminal organization and taking its internal structure as given. 
This paper is also related to the literature on social networks. In particular, Ballester et al. (2006), under the assumptions that the network structure is exogenously given and observed, characterize the "key player" - the player who, once removed, leads to the optimal change in aggregate activity. In this paper, instead, we start by asking how a network can be (endogenously) built to make criminal activity as efficient as possible.

\section{Model}

Suppose that there are $N>2$ members in a terrorist organization, with $N$ an even number and a single law enforcement agency. ${ }^{7}$ The law enforcement authority acts first and sets a given detection strategy as specified in Section 2.1. The $N$ terrorists have the possibility of forming an information structure by exchanging information among themselves as specified below in Section 2.2. ${ }^{8}$ After forming an information structure, the $N$ terrorists generate the benefits described in Section 2.2. Finally, detection takes place.

The assumption that the law enforcement authority chooses its policies before the terror structure is formed can be justified on the grounds that law enforcement policies and investigating budgets are broadly laid out and are hard to fine-tune once a certain policy is in place.

\subsection{Law Enforcement Agency}

At the end of the game, each terrorist can be detected by the enforcement agency. If the terrorist is detected, this imposes a direct cost to the organization of $k>0$. This cost may include a punishment for the individual, such as time in prison, and a cost to the organization of recruiting a new member.

There are two ways for a terrorist to be detected, a direct way and an indirect way. In particular, an independent Bernoulli random draw determines whether a particular terrorist is detected directly. The direct detection of a particular terrorist at each period is

\footnotetext{
${ }^{7}$ Allowing $N$ to be an odd number presents no conceptual difficulties, but adds to the number of cases that need be considered in some of the results with regard to how to treat the last odd agent, with no real gain in insight.

${ }^{8}$ We assume that the $N$ agents constitute an organization through some other production structure that is independent of the information structure. Although we do not explicitly model the formation process, one could assume that the information structure is determined by a "benevolent" third party. Indeed, this is essentially the approach advocated by Mustafa Setmariam Nasar, an Al-Qaeeda strategist who suggested that cell-builders be from outside the locale or immediately go on suicide missions after building cells.
} 
independent of other terrorists' detection. Thus, a terrorist $i$ can be detected directly by the authority according to some probability $\alpha_{i}$. This probability depends on the extent of the enforcement agency's scrutiny of terrorist $i$.

Second, the law enforcement authority might also detect terrorists indirectly. Indeed, we assume that when the agency detects a terrorist who has information about other members of the organization, the agency also detects each of these with probability $\gamma \in(0,1]$. First, note that this implies that the enforcement agency's ability to detect terrorists indirectly depends on the structure of the terror group. Second, $\gamma$ is a parameter that depends on the ability of the law enforcement agency to extract information from detected terrorists. For instance, the parameter $\gamma$ is determined by the interrogation methods and the ability to strike deals with prisoners in exchange for information.

The law enforcement agency has a budget $B \in[0, N]$ to allocate for investigating the $N$ members of the organization and devotes $\alpha_{i} \in[0,1]$ to investigating member $i$ where $\sum_{i=1}^{N} \alpha_{i} \leq B$. Without loss of generality, we label terrorists so that $\alpha_{1} \leq \alpha_{2} \leq \ldots \leq \alpha_{N}$. We refer to $\alpha_{i}$ as the enforcement agency's level of scrutiny (or investigation) of terrorist $i$.

\subsection{Information Structure}

We assume that each of the terrorists has a piece of private and verifiable information about himself and can decide to disclose this information to any of the other terrorists. Examples of such information could be the identity of the player, his whereabouts, some incriminating evidence, etc. We formalize the fact that $i$ holds such information about $j$ by an indicator variable $\mu_{i j}$, such that $\mu_{i j}=1$ if and only if $i$ knows the information regarding $j\left(\mu_{i j}=0\right.$ otherwise). We use the notation $j \rightarrow i$ to represent $\mu_{i j}=1$ (and, similarly, for instance $i, j, k \rightarrow l$ to represent $\left.\mu_{l i}=\mu_{l j}=\mu_{l k}=1\right) .{ }^{9}$ The set $\mathcal{I}$ of all the possible organization (or "information") structures among $N$ people is a subset of the set $\{0,1\}\}^{N^{2}}$ of values of the indicator variables, and we denote by $\mu$ its generic element.

We suppose that if $\mu_{i j}=1$ for some $i \neq j$ this yields a benefit $t$ for the organization. The idea here is that when someone in the organization holds information about $j$, it leads to more trust within the organization and, in particular, induces terrorist $j$ to cooperate with the organization's goals, and yielding a benefit $t$ to the organization. "Trust" and a benefit of a link in ensuring trust can be formalized in the context of cooperation in the

\footnotetext{
${ }^{9}$ Note that $\mu_{i i}=1$ for all $i$.
} 
infinitely-repeated Prisoners' dilemma - a standard approach in economics toolkit - and under the assumption that when $i$ has information about $j$ he can more easily punish him for not cooperating. A full discussion can be found in Baccara and Bar-Isaac (2008).

A terrorist $i$ is said to be indirectly linked to a terrorist $j$ if there is a path of direct links that connect $i$ to $j$. That is, if there is a set of terrorists $\left\{h_{1}, . ., h_{n}\right\}$ such that $i \rightarrow h_{1}$, $h_{1} \rightarrow h_{2}, . ., h_{n} \rightarrow j$.

The information structure affects the terrorists' probabilities of getting caught by the enforcement agency. Specifically, if $i$ has information about another member of the organization $j$, and $i$ is detected (either directly or indirectly), player $j$ is also detected with probability $\gamma$. As discussed in Section 2.1, if a terrorist $i$ is detected and there is a single path of length $d$ from terrorist $i$ to terrorist $j, j^{\prime} s$ probability of indirect detection as a result of $i$ 's capture is decaying in $d$ and, more precisely, is $\gamma^{d}$. Note, however, that there could be multiple paths from $i$ to $j$, and the total probability of detection of $i$ depends on all of them.

Recall from Section 2.1 that each terrorist $i$ is detected by the enforcement agency directly with probability $\alpha_{i}$ and indirectly as just discussed above. Overall, given an information structure $\mu$, let $\beta_{i}(\mu)$ be the total probability of detection of terrorist $i$.

In Figure 1, we give three examples of information structures. Panel A represents a decentralized structure in which terrorists are arranged in disconnected "cells" within which terrorists are linked to each other. Panel B represents a centralized structure (or "hierarchy"), in which terrorist 1 holds information on all other terrorists. Panel C represents a structure in which terrorists 1 and 2 form a cell, and the other two terrorists are connected to terrorist 1 in a hierarchical fashion.

If we assume that $\alpha_{i}=\alpha$ for $i=1, . ., 4$, in the information structure represented by Panel A in Figure 1, the probability of detection for each terrorist is $\beta_{i}(\mu)=\alpha+\alpha(1-\alpha) \gamma$ for $i=1, . ., 4$. The first term is the probability of direct detection, while the second term is the probability of indirect detection. Note that indirect detection occurs if the terrorist is not detected directly (with probability $(1-\alpha)$ ), the other member of the cell is caught directly (with probability $\alpha$ ), and the authority is able to extract information (with probability $\gamma)$.

In the information structure represented by Panel B in Figure 1, the probability of detection for terrorist 1 is $\alpha$, since terrorist 1 cannot be detected indirectly. However, $\beta_{i}(\mu)=\alpha+\alpha(1-\alpha) \gamma$ for $i=2,3,4$. Again, the first term is the probability of direct detection, while the second term is the probability of indirect detection. 


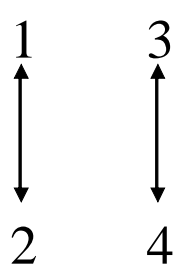

Panel A

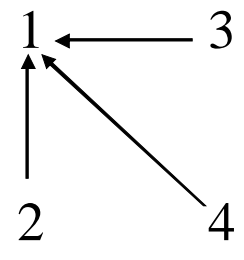

Panel B

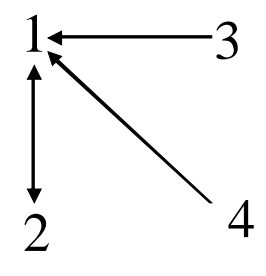

Panel C

Figure 1:

For the structure in Panel C, terrorists 1 and 2 form a cell, and their probability of detection is $\beta_{i}(\mu)=\alpha+\alpha(1-\alpha) \gamma$, while terrorists 3 and 4 can be detected if either terrorist 1 or 2 is detected. In particular, for $i=3,4$ we have

$$
\beta_{i}(\mu)=\alpha+(1-\alpha) \alpha \gamma+(1-\alpha)^{2} \alpha \gamma^{2}
$$

The first term of (1) is the probability of direct detection of terrorist $i$. The second term refers to the event in which terrorist 1 is detected directly and terrorist $i$ is not. In this case, terrorist $i$ is detected with probability $\gamma$. Finally, the third term represents the event in which terrorist 2 is detected directly, while terrorists 1 and $i$ are not. In this case, terrorist $i$ is detected with probability $\gamma^{2}$. (since terrorist 1 would first need to be detected as a result of terrorist 2's capture, and then the authority would need to extract the information from terrorist 1).

\subsection{Payoffs}

The payoff for the terror organization is given by the sum of the benefits generated by the links ( $t$ for every linked member), minus the expected cost of direct and indirect detection. Bringing together benefits and costs, we can write down the net payoffs to the organization of an information structure $\mu$ as:

$$
\Pi_{i}(\mu)=\operatorname{tn}(\mu)-\sum_{i=1}^{N} k \beta_{i}(\mu),
$$


where $n(\mu)$ denotes the number of members of the organization who are linked to some other member of the organization under the information structure $\mu$. Note that the total probability of detection $\beta_{i}(\mu)$ depends on the probabilities of direct detections $\left\{\alpha_{j}\right\}$, the information structure $\mu$, and the decay parameter $\gamma$.

We assume that the goal of the enforcement agency is to minimize the trust among the $N$ terrorists. In other words, to minimize $n(\mu)$ and the benefits that the terror organization obtains from linking its members to each other. For simplicity, we assume that the authority gets no utility from saving part of the budget $B$. Also, the enforcement agency does not benefit from the the payments $k$ incurred by the detected terrorists. ${ }^{10}$

Baccara and Bar-Isaac (2008) provides some further justification and extensive discussion on the benefits of links and on potential extensions and developments of the model.

\section{The Optimal Network}

In this section, we study how a strategic terror organization chooses its structure, given that a specific enforcement policy is in place. In particular, we take the authority's scrutiny $\left\{\alpha_{1}, \ldots, \alpha_{N}\right\}$ as given, and we study the most efficient information structure that the $N$ terrorists can form. These characterizations will allow us to tackle the problem of the government's optimal behavior. ${ }^{11}$

In characterizing the optimal information structures, it is useful to begin by focusing on optimal structures given a fixed number of terrorists "linked" to other terrorists - that is, a fixed number of terrorists that disclose their information to at least one other terrorist. Note that the benefits of having terrorists linked depend only on their number rather than on the structure of the organization. In particular, the potential benefit that the organization can yield from the links is constant with respect to all the information structures with the same number of terrorists linked to someone else. Thus, if the number of linked terrorists is fixed, an optimal organization minimizes the cost of information leakage.

We begin by characterizing the optimal information structure when the number of

\footnotetext{
${ }^{10}$ Indeed, these payments may be costly for the enforcement agency. For example, they may consist of detention in prison facilities.

${ }^{11}$ Note that even though we assume that the enforcement agency determines the level of scrutiny, these probabilities could also be exogenously given and due to some intrinsic characteristics of the agents. For example, some members of the organization may be more talented in evading detection (some may have a cleaner criminal record or simply might be able to run faster). If this is the case, the optimal organization characterizations we provide in this section can be seen as self-contained.
} 
linked terrorists $n$ is strictly less than $N$ in the next Proposition.

Proposition 1 The optimal structure to link $n<N$ terrorists is a hierarchy with the terrorist with the lowest probability of detection at the top, and the $n$ terrorists with the highest probabilities of detection linked to him (i.e., $N, N-1, \ldots, N-n+1 \longrightarrow 1$ ).

If the number of linked terrorists is less than $N$, the optimal structure is simply a hierarchy in which the top (the terrorist who receives the information from the others) is the member with the lowest probability of detection and the $n<N$ "linked" terrorists are those with the $n$ highest probability of detection. The proof of Proposition 1 is very simple. Recall that, without loss of generality, we have $\alpha_{1} \leq \alpha_{2} \leq \ldots \leq \alpha_{N}$. Suppose, first, that $n=1$, so we need to find the way to generate the "cheapest" possible link in terms of information leakage costs. The only event in which this link becomes costly is the case in which member $i$ is independently detected and member $j$ is not. This event has probability $\alpha_{i}\left(1-\alpha_{j}\right)$. Then, the cost of the link is minimized when $\alpha_{i}$ is as small as possible and $\alpha_{j}$ is as large as possible. It follows that the "cheapest" possible link is the one that requires member $N$ to disclose his information to member 1 (the link $N \rightarrow 1$ ). If $n=2$, the second cheapest link one can generate after $N \rightarrow 1$ is $N-1 \rightarrow 1$, and so on. Notice that Proposition 1 implies that the information leakage cost under an optimal structure in which there are $n<N$ links is simply $k \alpha_{1} \gamma \sum_{i=1}^{n}\left(1-\alpha_{N-i+1}\right)+k \sum_{i=1}^{N} \alpha_{i}$.

Next, consider the case where all $N$ terrorists are linked to someone else. To start the characterization, consider a cell $\{i, j\}$. Let $\rho(i, j) \equiv \frac{\alpha_{i}+\alpha_{j}-2 \alpha_{i} \alpha_{j}}{2-\alpha_{i}-\alpha_{j}}$. This is a useful ratio in understanding the organization members' proclivity to be linked as a binary cell rather than as subordinates to another terrorist. If two members $\{i, j\}$ are in a cell, terrorist $i$ will get caught and the organization suffers the cost $k$ with probability $\alpha_{i}+\left(1-\alpha_{i}\right) \alpha_{j} \gamma$. However, if each of them is independently linked to a third terrorist (the same for both, and who may be linked to others) with overall probability of detection $\eta$, terrorist $i$ will get caught and the organization will suffer a cost $k$ with probability $\alpha_{i}+\eta \gamma\left(1-\alpha_{i}\right)$, and terrorist $j$ will get caught with probability $\alpha_{j}+\eta \gamma\left(1-\alpha_{j}\right)$. Then, having the terrorists $\{i, j\}$ forming an independent cell rather than linking each of them to the third terrorist minimizes the cost of information leakage if and only if

$$
\alpha_{i}+\left(1-\alpha_{i}\right) \alpha_{j} \gamma+\alpha_{j}+\left(1-\alpha_{j}\right) \alpha_{i} \gamma<\alpha_{i}+\eta \gamma\left(1-\alpha_{i}\right)+\alpha_{j}+\eta \gamma\left(1-\alpha_{j}\right)
$$


or, equivalently,

$$
\rho(i, j)=\frac{\alpha_{i}+\alpha_{j}-2 \alpha_{i} \alpha_{j}}{2-\alpha_{i}-\alpha_{j}}<\eta .
$$

Thus, for any couple of terrorists, the higher is $\rho(i, j)$, the greater is the advantage of forming a cell rather than being linked to a third terrorist. Note that $\rho(i, j)$ is decreasing in both $\alpha_{i}$ and $\alpha_{j}$-that is, the higher the probability of detection of a terrorist, the lower $\rho(i, j)$ of any cell to which he might belong. Note, also, that $\rho(i, j)$ does not depend on $\gamma$. This is because decaying probabilities of detection affect the optimality of independence for a cell only if linking themselves to a third terrorist creates indirect links in the organization - that is, if it affects the probability $\eta$.

We now characterize the optimal information structure with $N$ linked terrorists in the following proposition.

Proposition 2 The optimal information structure with $N$ linked terrorists is described as follows. Let $i^{*} \in\{2, . ., N\}$ be the largest even integer such that $\rho(i-1, i)>\alpha_{1}+\left(1-\alpha_{1}\right) \alpha_{2} \gamma$ (if no such integer exists, set $i^{*}=1$ ): All the terrorists $i=1, . ., i^{*}$ are arranged in binary cells as $1 \leftrightarrow 2,3 \longleftrightarrow 4, . ., i^{*}-1 \longleftrightarrow i^{*}$, and the terrorists $i=i^{*}+1, . ., N$ all reveal their information to terrorist 1 , that is, $i^{*}+1, . ., N \rightarrow 1$.

Proposition 2 states that the optimal way to link $N$ members in a terror organization is to divide the members into two groups according to their probabilities of detection: a group comprising the $i^{*}$ members with the lowest probabilities of detection, and another group with the $N-i^{*}$ members with the highest probability of detection. The members belonging to the first group are arranged in binary cells formed by members with adjacent probability of detection (i.e. $1 \leftrightarrow 2,3 \longleftrightarrow 4, . ., i^{*}-1 \longleftrightarrow i^{*}$ ). All the members belonging to the second group reveal their information to terrorist $2\left(i^{*}+1, . ., N \rightarrow 1 \leftrightarrow 2\right)$.

The number of terrorists $i^{*}$ belonging to the independent cell component depends on how steeply the ratio $\rho(i, i+1)$ of each couple grows. If $\alpha_{1}$ and $\alpha_{2}$ are very low relative to the other terrorists' probabilities of detection, it could be the case that $\rho(i-1, i)>$ $\alpha_{1}+\left(1-\alpha_{1}\right) \alpha_{2} \gamma$ for all $i=4, . ., N$. In this case, Proposition 2 requires that an optimizing organization links all the members $3, . ., N$ to terrorist 1 (who remains linked in a cell with 2). ${ }^{12}$ However, if $\alpha_{3}$ and $\alpha_{4}$ are close enough to $\alpha_{2}$, then $\rho(3,4)>\alpha_{2}+\left(1-\alpha_{2}\right) \alpha_{1} \gamma$, and

\footnotetext{
${ }^{12}$ In particular, if $\alpha_{1}$ and $\alpha_{2}$ approach zero, all these links have an arbitrarily small information leakage
} 


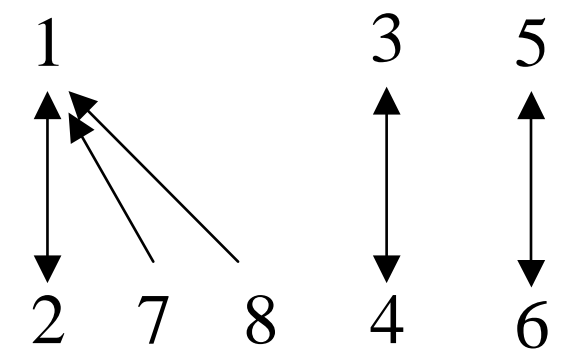

Figure 2:

Proposition 2 prescribes 3 and 4 to form a cell rather than being linked to 1 , and so on.

The optimal information structure described in Proposition 2 is illustrated in Figure 2, when there are $N=8$ terrorists and $i^{*}=6$.

Note that with a full characterization of the form of optimal structures, we can easily calculate the information leakage of linking $n$ terrorists and inducing them to cooperate. Comparing costs and benefits of links then allows us to characterize the optimal information structure.

Lemma 1 and Proposition 2 allow us to define the net benefit to the organization of having $n$ links. In particular, if the organization has $N$ links, this yields a direct "trust" benefit of $N t$, a direct cost of $k \sum_{i=1}^{N} \alpha_{i}$ of detection, and a cost of indirect detection: For a terrorist $i$ (from $i^{*}+1$ to $N$ ) who is subordinate to the $1 \longleftrightarrow 2$ informational hub, the cost of indirect detection is $k\left(\alpha_{1} \gamma+\alpha_{2}\left(1-\alpha_{1}\right) \gamma^{2}\right)\left(1-\alpha_{i}\right)$, whereas for a terrorist $2 i$ in a cell with terrorist $2 i-1$, the cost of indirect detection is $k \gamma\left(1-\alpha_{2 i}\right) \alpha_{2 i-1}$. Summing all benefits and costs over all terrorists, we can obtain that the value to an organization of having all $N$ terrorists linked (in the most efficient way) is:

$$
V(N)=\begin{aligned}
& N t-k \sum_{\substack{i=1 \\
\frac{i^{*}}{2}}}^{N} \alpha_{i}-k\left(\alpha_{1} \gamma+\alpha_{2}\left(1-\alpha_{1}\right) \gamma^{2}\right) \sum_{i=i^{*}+1}^{N}\left(1-\alpha_{i}\right) \\
& -k \gamma \sum_{i=1}^{2}\left[\left(1-\alpha_{2 i-1}\right) \alpha_{2 i}+\left(1-\alpha_{2 i}\right) \alpha_{2 i-1}\right]
\end{aligned}
$$

cost, so the organization's information leakage cost is the same as in the structure with no links. 
Similarly we can write $V(n)=n t-k \sum_{i=1}^{N} \alpha_{i}-k \gamma \alpha_{1} \sum_{j=N-n+1}^{N}\left(1-\alpha_{j}\right)$ for $n \in\{1, \ldots, N-1\}$ and $V(0)=-k \sum_{i=1}^{N} \alpha_{i}$

With expressions for the value of having $n$ links, we can, therefore, find the number of links $n^{*}$ that maximizes the value of the organization, $V(n)$. The following proposition follows trivially from the earlier results and characterizes the optimal information structure.

Proposition 3 If $\gamma=1$, the optimal information structure is as follows: (i) If $n^{*}=0$, the optimal information structure is an anarchy. (ii) If $0<n^{*}<N$, the optimal structure is an individual-dominated hierarchy where the hub is terrorist 1 and the subordinates are terrorists $N, \ldots N-n+1$. (iii) Finally, if $n^{*}=N$, the optimal structure is the mixed structure described in Proposition 2.

Note that when $\alpha_{i}=\alpha$ for all $i$, the additional cost of each link is constant and equal to $k \gamma \alpha(1-\alpha)$. It follows that, in this symmetric case, the optimal structure either consists of no links or all terrorists are in binary cells.

Corollary 4 Let $\alpha_{i}=\alpha$ for all $i$. If $t>k \gamma \alpha(1-\alpha)$, then the optimal information structure is a binary cell structure. Otherwise, the optimal information structure has no links.

This concludes the characterization of the optimal information structure for a given scrutiny distribution $\left\{\alpha_{1}, . ., \alpha_{N}\right\}$. Next, we endogenize scrutiny and discuss the strategy of the enforcement agency.

\section{The Enforcement Agency}

The enforcement agency aims to minimize production within the criminal organization. To do so, the agency has several instruments to affect how criminal organizations operate. First, as discussed in Section 2.1, the enforcement agency has a budget $B$ to allocate to detect terrorists.

Second, two further parameters in our model affect the enforcement agency's ability to minimize cooperation within the organization. These parameters are $\gamma$, which measures how effective the interrogation techniques are in extracting information from detected terrorists, and $k$, which measures the severity of the punishment for detected terrorists. Note 
that both these parameters are typically constrained by legal limits. Thus, the parameters $(\gamma, k)$ can be viewed as describing the legal environment in which authorities act and the effectiveness of their interrogation methods.

In this section, first we study how these three instruments should be used and how they are interrelated. In particular, we first show how, for any fixed legal environment $(\gamma, k)$, an authority should optimally allocate its investigation budget to minimize cooperation within the organization - that is, to minimize the number of linked terrorists. Next, we look at how a criminal organization is likely to strategically respond to changes of $\gamma$ and $k$ - that is, changes in the legal environment - and, in turn, how the optimal investigation budget allocation should be optimally altered in response to such legal changes.

\subsection{Investigation Budget Allocation}

As discussed in Section 2.3, we assume that the enforcement agency's objective is to minimize the number of terrorists who cooperate - that is, the organization's production level $n^{*} t$, where $n^{*}$ maximizes the value of the organization, $V(n)$.

Recall that, for a given legal environment $(\gamma, k)$, the problem of the law enforcement agency is to allocate the investigation budget $B \in[0, N]$ to determine the scrutiny $\alpha_{i} \in[0,1]$ of each terrorist $i$ such that $\sum_{i=1}^{N} \alpha_{i} \leq B$. The enforcement agency acts first and chooses these scrutiny levels before the organization forms.

In the next result, we characterize the (weakly) optimal strategy for the enforcement agency in allocating its resources. Note that, if the authority allocates the same budget $\alpha$ to each terrorist, the cost of each link becomes $k \gamma \alpha(1-\alpha)$. Since this cost is maximized at $\alpha=\frac{1}{2}$, it is never optimal to set $\alpha>\frac{1}{2}$ in a symmetric allocation. Let, then, $\widehat{\alpha}=\min \left\{\frac{B}{N}, \frac{1}{2}\right\}$ be the optimal symmetric allocation.

Proposition 5 A weakly optimal strategy for the enforcement agency is to set scrutiny symmetrically if $k \gamma \widehat{\alpha}(1-\widehat{\alpha})>t$ and to not investigate one terrorist and investigate all others symmetrically (set $\alpha_{1}=0$ and $\alpha_{2}=\ldots=\alpha_{N}=\min \left\{\frac{B}{N-1}, 1\right\}$ ) otherwise.

A symmetric allocation of $B$ can prevent the formation of any link if the cost of each link $k \gamma \widehat{\alpha}(1-\widehat{\alpha})$ is greater than the benefit of inducing an individual to cooperate. This is the case when $k \gamma \widehat{\alpha}(1-\widehat{\alpha})>t$, and, in these circumstances, a symmetric allocation is optimal as it deters any cooperation. 
However, if $k \gamma \widehat{\alpha}(1-\widehat{\alpha})<t$, by Lemma 4, a symmetric allocation would imply the formation of a binary cell structure that reaches full cooperation within the organization. The question is whether, in these situations, the enforcement agency can do something else to prevent full cooperation. Proposition 5 addresses this question and suggests that, in this case, an allocation in which one terrorist remains undetected and the budget is equally divided among the other $N-1$ terrorists is optimal. Under this allocation, sometimes the organization still reaches full efficiency (in this case, we can conclude that the enforcement agency cannot prevent full efficiency for the terror network), but in some cases, a hierarchy with $N-1$ links arises. Since the hierarchy is strictly less efficient than a binary cell structure, this allocation strictly dominates the symmetric one.

If $k \gamma \widehat{\alpha}(1-\widehat{\alpha})>t$, we show that there is no other allocation that strictly dominates $\alpha_{1}=0$ and $\alpha_{2}=\ldots=\alpha_{N}=\min \left\{\frac{B}{N-1}, 1\right\} .{ }^{13}$ The intuition for this part of Proposition 5 is the following. First of all, note that if two terrorists remain undetected $\left(\alpha_{1}=\alpha_{2}=0\right)$, the organization can form $N$ links without incurring any additional information leakage costs with respect to the cost they would incur with no links (this is because the two terrorists can reveal information to each other at no cost and costlessly act as a hub for the $N-2$ terrorists). So, to deter full efficiency, the enforcement agency can leave, at most, one terrorist undetected. Suppose now that some cooperation is deterred by an allocation in which all terrorists are detected with some probability $\left(\alpha_{1}>0\right)$. Then, the terrorist with the lowest allocation will act as a hub in a hierarchy, as described in Proposition 1. In the Appendix, we prove that under our assumption, there are exactly $N-1$ links in such a hierarchy. Then, moving all the resources from the hub to the other terrorists, as suggested in Proposition 5, is equivalent to the original allocation.

Let us turn to comment on the cooperation or trust outcomes in equilibrium. Proposition 5 states that in some circumstances (i.e., if $k \gamma \widehat{\alpha}(1-\widehat{\alpha}) \geq t$ ), the enforcement agency can prevent any cooperation in the organization by allocating its budget symmetrically. Note that these circumstances are more likely to occur when $k$ is higher, or the benefit from trust $t$ is lower. However, a higher budget $B$ is beneficial as long as it is below the threshold $\frac{N}{2}$. Increases in $B$ beyond that threshold would have no further effect on the cooperation level in the organization. On the other hand, when $k \gamma \widehat{\alpha}(1-\widehat{\alpha})<t$, the optimal detection strategy for the authority deters, at most, one link and the trust in or cooperation of one terrorist in the organization.

\footnotetext{
${ }^{13}$ Note that considering $\min \left\{\frac{B}{N-1}, 1\right\}$ guarantees that the resulting allocation on each agent is in the interval $[0,1]$.
} 


\subsection{Legal Environment and Interrogation Methods}

Two parameters in our model directly describe the legal environment within which the authorities act. In particular, the parameter $\gamma$ measures the probability with which an agent in the hands of the authority reveals the information he knows, and the parameter $k$ measures the severity of the punishment inflicted on the detected agents. Thus, a broadening of the admissible measures (such as, for example, the Patriot Act) can be captured in this model as an increase of $\gamma$ or $k$, or both.

In this section, we look at how a criminal organization is likely to strategically respond to changes of $\gamma$ and $k$ and, in turn, how effective such changes are and which investigation budget allocation should be optimally associated with them.

First, let us look at how, given a budget detection allocation $\left\{\alpha_{1}, \ldots, \alpha_{N}\right\}$, a change in the legal environment affects the optimal information structure. Examples of change in legal environments include banning the use of torture, and allowing reduced sentences and rewards for whistle-blowers.

From the results in Section 3, the effect of changes in $\gamma$ on the optimal information structure is described in the following Proposition.

Proposition $6 \quad$ 1. If $\gamma<\underline{\gamma}$, the optimal organization structure is a mixed strategy as described in Proposition 2, where the number of binary cells in the structure is increasing in $\gamma$.

2. If $\underline{\gamma}<\gamma \leq \bar{\gamma}$, a simple hierarchy as described in Proposition 1 is optimal. The number of linked agent is decreasing in $\gamma$.

3. If $\bar{\gamma}>\gamma$, the optimal information structure has no links.

Proposition 6 suggests that for low levels of $\gamma$, an increase in $\gamma$ does not have the effect of decreasing cooperation within the organization, but has the effect of increasing decentralization. However, sufficiently high increases in $\gamma$ reduce the number of linked terrorists and make a simple hierarchy optimal.

To understand the argument behind point (1) of Proposition 6, recall from Proposition 2 that the optimal number of terrorists organized in cells is found by defining $i^{*} \in\{2, . ., N\}$ as the largest even integer such that $\rho(i-1, i)>\alpha_{1}+\left(1-\alpha_{1}\right) \alpha_{2} \gamma$. Since $\rho(i-1, i)$ is independent of $\gamma$, an increase in $\gamma$ increases $i^{*}$ and, thus, the number of resulting cells. 
Proposition 6 has significant policy implications. Suppose that an asymmetric detection strategy is in place, and the criminal organization responds with a partially centralized structure, as described in Proposition 2. In such a structure, there are two agents (i.e., agents 1 and 2) who, directly or indirectly, hold a significant amount of information about other members of the organization. Thus, authorities may expect that toughening the interrogation methods (as, for example, by allowing the use of torture) will lead to more information being revealed following capture of such a critical member of the terror organization. However, our approach views the terror network as a strategic agent that will optimally respond to such environmental change. In particular, Proposition 6 suggests that there are circumstances under which the toughening of interrogation methods will fail to achieve less cooperation within the organization, and it will simply lead to more decentralization in the way information is shared among the members.

Next, we consider how an increase in the severity of the punishment (measured in our model by $k$ ) affects the optimal structure of the organization.

Proposition $7 \quad$ 1. If $k<\underline{k}$, a mixed structure as described in Proposition 2 is optimal. The number of binary cells in the structure is constant in $k$.

2. If $\underline{k} \leq k<\bar{k}$, a simple hierarchy as described in Proposition 1 is optimal. The number of linked agents is decreasing in $k$.

3. If $k>\bar{k}$, the optimal information structure has no links.

Propositions 6 and 7 suggest that, while a change in the interrogation methods (i.e., a change in $\gamma$ ) can lead to a more decentralized organization, a change in the severity of the punishment (i.e., a change in $k$ ) never has this effect.

Next, we look at how the enforcement agency should optimally adjust the optimal detection strategy $\left\{\alpha_{1}, . ., \alpha_{N}\right\}$ after a change in the legal environment $(\gamma, k)$.

Proposition 8 Following an increase of $\gamma$ or $k$, a symmetric investigation budget allocation is more likely to be optimal.

The intuition behind Proposition 8 is simple. Given an investigation budget $B$, by broadening the legal limits and increasing $\gamma$ or $k$ (or both), the cost to the terror organization of a link in the binary cell structure increases. This makes it more likely that 
any cooperation within the organization can be prevented by a symmetric allocation of the budget $B$.

Although Proposition 8 is a direct consequence of Proposition 5, it offers interesting insights from a policy perspective. Indeed, this result suggests that the three instruments available to the authorities (investigation budget allocation, interrogation methods and severity of punishments) are not independent but are strategically interrelated. Thus, any change in the legal environment should be associated with a revised investigation budget allocation. In particular, if the legal environment broadens the legal limits within which the authorities can act (i.e., an increase in $\gamma$ and $k$ ), a symmetric budget allocation is more likely to be optimal than before. However, if the legal limits for the authorities become narrower, it is more likely that a symmetric allocation will lead to a fully linked binary cell structure. Thus, the authorities could do better by implementing an asymmetric detection strategy as described in Proposition 5.

\section{Extensions and Conclusions}

In the framework outlined above, we assumed that the law enforcement authority acts first, and the terrorist organization chooses a structure in response. Another possibility is that the authority might choose its strategy first, the organization then forms, and the authority's strategy is then realized. If the authority is restriced to choose a pure strategy, this timing assumption leads to results equivalent to this paper's. However, if the authority can choose mixed strategies then this timing assumption is substantive and can lead to more effective strategies for the enforcement agency. For example, suppose that there are only two agents in the organization. Randomizing between choosing scrutinies of $(0,1)$ and $(1,0)$ is more likely to the deter creation of a binary cell than a symmetric allocation of $\left(\frac{1}{2}, \frac{1}{2}\right)$. However, in the case where there are many agents, then a symmetric and deterministic allocation might be more likely to deter the creation of $N$ or $N-1$ links than mixing asymmetric ones.

Numerous extensions to framework above might be considered. Baccara and Bar-Isaac (2008) provides a more careful consideration of the benefits of informational links and it considers a model where the a terrorist's behavior can depend on his position in the structure, and the probability of getting caught depends on behavior. Other potentially interesting extensions of this framework include exploring the process of the formation of the structure, and a more detailed and careful consideration of the costs to the organization 
of having members detected. In all these cases, the fundamental points will still apply: The structure of a terrorist network responds (in a way that can be characterized) to law enforcement policies, and these policies should be tailored to the legal environment.

\section{References}

[1] Anselmi T. (2003), "La testimonianza di Tina Anselmi sulla Resistenza raccolta da Alessandra Chiappano," http://www.novecento.org.

[2] Arquilla J. and D. F. Ronfeldt (2002), "Networks and Netwars: The Future of Terror, Crime, and Militancy" Rand Corporation.

[3] Aubert C., Kovacic W. and Rey P. (2006), "The impact of leniency and whistle-blowing programs on cartels," International Journal of Industrial Organization, Volume 24, Issue 6, November 2006, Pages 1241-1266.

[4] Baccara, M. and H. Bar-Isaac (2008), "How to Organize Crime," The Review of Economic Studies, forthcoming.

[5] Bandiera O. (2003), "Land Reform, the Market for Protection and the Origin of Sicilian Mafia," Journal of Law, Economics and Organizations, Vol.19.

[6] Ballester, C., Calvó-Armengolz A., and Zenou, Y. (2006) "Who's Who in Networks. Wanted: The Key Player," Econometrica, Vol.74, 1403-1418.

[7] Charlie M. (2002) "Into the Abyss: A Personal Journey Into the World of Street Gangs", Manuscript.

[8] Dixit, A. (2004), Lawlessness and Economics: Alternative Modes of Governance, Princeton University Press, Princeton NJ

[9] Falcone G. (1991), Cose di Cosa Nostra, Rizzoli, Milan.

[10] Falcone G. and G.Turone (1982), "Tecniche di Indagine in Materia di Mafia," Report to the consiglio Superiore della Magistratura.

[11] Farley, J. D. (2003), "Breaking Al Qaeda Cells: A Mathematical Analysis of Counterterrorism Operations (A Guide for Risk Assessment and Decision Making)," Studies in Conflict and Terrorism, Vol. 26, 399-411. 
[12] Farley, J. D. (2006), "Building the perfect terrorist cell," conference presentation.

[13] Gambetta D. (1993), The Sicilian Mafia, Harvard University Press, Cambridge, Mass.

[14] Garoupa, N. (2007), "Optimal law enforcement and criminal organization," Journal of Economic Behavior \& Organization, 63(3), 461-474.

[15] Garreau, J. (2001), "Disconnect the Dots," Washington Post, September 16, 2001.

[16] Harrington J. (2003), "Some Implications of Antitrust Laws for Cartel Pricing," Economics Letters, Vol. 79, June 2003, 377-383.

[17] Harrington J. (2004), "Cartel Pricing Dynamics in the Presence of an Antitrust Authority," RAND Journal of Economics, Vol. 35, Winter 2004, 651-673.

[18] Harrington J. (2005), "Optimal Cartel Pricing in the Presence of an Antitrust Authority," International Economic Review, Vol. 46, February 2005, 145-169.

[19] Harrington, J. (forthcoming): "Optimal Corporate Leniency Programs," Journal of Industrial Economics, forthcoming.

[20] Maskin E., Y. Qian, C. Xu (2000), "Incentives, Information, and Organizational Form," The Review of Economic Studies, Vol. 67, No. 2, 359-378.

[21] Oliva A. (1976), "Esercito and Democrazia", Vangelista Editore.

[22] Pearl M., (2003), "A Mighty Heart: The Inside Story of the Al Qaeda Kidnapping of Danny Pearl", Scribner Publishing.

[23] Schulhofer S. J. (2005), Rethinking the Patriot Act: Keeping America Safe and Free, Century Foundation Press.

[24] Smith A. and F. Varese (2001,) "Payment, Protection and Punishment," Rationality and Society, Vol. 13(3), 349-393.

[25] Spagnolo, G. (2003), "Optimal Leniency Programs," Mimeo

[26] Spagnolo, G. (2007): "Leniency and Whistleblowers in Antitrust," forthcoming as Ch. 12 of P. Buccirossi (Ed.), Handbook of Antitrust Economics, 2007, M.I.T. Press.

[27] Stille A. (1995), Excellent Cadavers, Pantheon Books, New York, NY. 
[28] Thompson, T. (2005), Gangs: A Journey into the Heart of the British Underworld, Hodder \& Stoughton.

[29] Tucker, C. (2007): "Formal and Informal Influence in the Adoption of Technologies with Network Effects" working paper

[30] Williamson O. E. (1983), "Credible Committments: Using Hostages to Support Exchange," American Economic Review, vol.73(4).

[31] Zabojnik J. (2002), "A Theory of Trade Secrets in Firms," International Economic Review, vol.43.

\section{A Appendix}

Proof of Proposition 2 First step. Recall that $\rho(j, i)$ is decreasing in both $\alpha_{j}$ and $\alpha_{i}$. This follows easily by taking the derivative of $\rho(i . j)$ with respect to $\alpha_{i}$ and $\alpha_{j}$ and noting that $0 \leq \alpha_{i}, \alpha_{j} \leq 1$.

Second step Let us prove that among all possible binary cell information structures that pair $N$ terrorists to each other $\left\{\mu \in I\right.$ s.t. if $\mu_{i j}=1$ for some $i \neq j$ then $\mu_{j i}=1$ and $\left.\mu_{i k}=0 \forall k \neq j\right\}$, the one which minimizes information leakage costs is $1 \longleftrightarrow 2,3 \longleftrightarrow 4, \ldots, N-1 \longleftrightarrow N$. To see this, let us first show that this result holds for $N=4$. The claim is true if $1 \longleftrightarrow 2,3 \longleftrightarrow 4$ is better than either of the alternatives $1 \longleftrightarrow 4,2 \longleftrightarrow 3$ and $1 \longleftrightarrow 3,2 \longleftrightarrow 4$. This requires that:

$$
\begin{aligned}
& k\left[\alpha_{1}+\left(1-\alpha_{1}\right) \alpha_{2} \gamma+\alpha_{2}+\left(1-\alpha_{2}\right) \alpha_{1} \gamma\right]+k\left[\alpha_{3}+\left(1-\alpha_{3}\right) \alpha_{4} \gamma+\alpha_{4}+\left(1-\alpha_{4}\right) \alpha_{3} \gamma\right] \leq \\
& k\left[\alpha_{1}+\left(1-\alpha_{1}\right) \alpha_{4} \gamma+\alpha_{4}+\left(1-\alpha_{4}\right) \alpha_{1} \gamma\right]+k\left[\alpha_{2}+\left(1-\alpha_{2}\right) \alpha_{3} \gamma+\alpha_{3}+\left(1-\alpha_{3}\right) \alpha_{2} \gamma\right]
\end{aligned}
$$

and,

$$
\begin{aligned}
& k\left[\alpha_{1}+\left(1-\alpha_{1}\right) \alpha_{2} \gamma+\alpha_{2}+\left(1-\alpha_{2}\right) \alpha_{1} \gamma\right]+k\left[\alpha_{3}+\left(1-\alpha_{3}\right) \alpha_{4} \gamma+\alpha_{4}+\left(1-\alpha_{4}\right) \alpha_{3} \gamma\right] \leq \\
& k\left[\alpha_{1}+\left(1-\alpha_{1}\right) \alpha_{3} \gamma+\alpha_{4}+\left(1-\alpha_{3}\right) \alpha_{1} \gamma\right]+k\left[\alpha_{2}+\left(1-\alpha_{2}\right) \alpha_{4} \gamma+\alpha_{4}+\left(1-\alpha_{4}\right) \alpha_{2} \gamma\right]
\end{aligned}
$$

Inequality (6) holds if $\alpha_{1} \alpha_{2}+\alpha_{3} \alpha_{4} \geq \alpha_{1} \alpha_{4}+\alpha_{2} \alpha_{3}$ or if $\left(\alpha_{4}-\alpha_{2}\right)\left(\alpha_{3}-\alpha_{1}\right) \geq 0$, which is always the case. Inequality (7) also always holds.

Now, suppose that for a general even $N$ the claim is not true. Then, there is an optimal structure in which it is possible to find 2 pairs $\left\{i_{1}, i_{2}\right\},\left\{i_{3}, i_{4}\right\}$ such that $\alpha_{i_{1}} \leq \alpha_{i_{2}} \leq \alpha_{i_{3}} \leq \alpha_{i_{4}}$ is violated. Then, since that is the optimal structure, rearranging the terrorists in these pairs leaving all other pairs unchanged cannot reduce information leakage costs. However, this contradicts the result for $N=4$.

Third step. It is clear that the best way to link terrorists 1 and 2 is to link them to each other since they are the two lowest-probability terrorists. Now, for any couple $\{N-1, N\}, \ldots,\{3,4\}$ let us compare whether it is better from an information leakage point of view to link the pair to each other and independently from the others, or to have them linked to terrorist 1 (and 2) instead. If the terrorists $N$ and $N-1$ are linked to each other, the cost of information leakage corresponding to the couple is $k\left[\alpha_{N-1}+\left(1-\alpha_{N-1}\right) \alpha_{N} \gamma+\alpha_{N}+\left(1-\alpha_{N}\right) \alpha_{N-1} \gamma\right]$. If they are linked to terrorists 1 and 2 , 
the cost of information leakage is $k\left[\alpha_{N-1}+\left(1-\alpha_{N-1}\right)\left(\alpha_{1}+\alpha_{2}\left(1-\alpha_{1}\right) \gamma\right) \gamma+\alpha_{N}+\left(1-\alpha_{N}\right)\left(\alpha_{1}+\alpha_{2}\left(1-\alpha_{1}\right) \gamma\right) \gamma\right]$. Then, the couple $\{N-1, N\}$ should be linked to terrorist 1 (and then, since we have $1 \leftrightarrow 2$, to the couple $\{1,2\}$ ) if and only if

$$
\rho(N-1, N)<\left(\alpha_{1}+\alpha_{2}\left(1-\alpha_{1}\right) \gamma\right) \gamma
$$

If condition (8) fails, by the first step of this proof we know that the condition will fail for any subsequent couple. Then, the optimal way to link the $N$ terrorists to each other is to create a pairwise structure and, by the second step of this proof, we know that the optimal way to do this is to set $1 \leftrightarrow 2,3 \longleftrightarrow 4, .$. and $N \longleftrightarrow N-1$. If condition (8) is satisfied, we can link terrorists $N$ and $N-1$ to the couple $\{1,2\}$, and we can repeat this check for the couple $\{N-2, N-3\}$. We repeat this process until we find a couple $\{i-1, i\}$ for which the condition

$$
\rho(i-1, i)<\left(\alpha_{1}+\alpha_{2}\left(1-\alpha_{1}\right) \gamma\right) \gamma
$$

fails. If we find such a couple, by the first step of this proof we know that the condition will fail for any subsequent couple, and, by the second step of the proof, we can arrange any subsequent couple in a pairwise fashion.

Proof of Proposition 5 In order to prove this result, we prove the following Lemma first. Let $\widetilde{\alpha} \equiv\left\{0, \frac{B}{N-1}, . ., \frac{B}{N-1}\right\}$.

Lemma The allocation $\widetilde{\alpha}$ minimizes the net benefit of the Nth link (linking terrorist 1 to terrorist 2) compared to any other allocation $\alpha$ which generates exactly N-1 links.

Proof: Consider any allocation $\alpha$ that generates exactly $N-1$ links. Since $\alpha_{1} \leq \alpha_{2} \leq \ldots \leq \alpha_{N}$ and $\alpha_{1} \geq 0$, it follows that $\alpha_{2} \leq \frac{B}{N-1}$. We can compare the additional information leakage costs from the $N-$ th link, $V(N)=V(N)-V(N-1)$ and $\widetilde{V}(N)=\widetilde{V}(N-1)-\widetilde{V}(N-1)$ associated with each terrorist $i$ under allocations $\alpha$ and $\widetilde{\alpha}$. In order to do that, let us consider the allocation $\widehat{\alpha} \equiv\left\{0, \alpha_{2}, . ., \alpha_{N}\right\}$ and first compare $\alpha$ with $\widehat{\alpha}$. Under the optimal information structures with $N$ links described in Proposition 2, given allocation $\alpha$, either (a) terrorist $i$ remains linked to terrorist 1 or (b) terrorist $i$ is in a binary cell with some other terrorist $j$ in the organization (which will be $i+1$ or $i-1$ depending on whether $i$ is even or odd). In case (a), the incremental leakage cost for terrorist $i$ is $k \gamma^{2}\left(1-\alpha_{i}\right)\left(1-\alpha_{1}\right) \alpha_{2}$, while under allocation $\widehat{\alpha}$ is going to be $k \gamma^{2}\left(1-\alpha_{i}\right) \alpha_{2}$. Trivially, $k \gamma^{2}\left(1-\alpha_{i}\right)\left(1-\alpha_{1}\right) \alpha_{2}<k \gamma^{2}\left(1-\alpha_{i}\right) \alpha_{2}$. In case (b), since the incremental information leakage cost for terrorists $i$ and $i+1$ of the $N-t h$ link under allocation $\alpha$ is $k \gamma\left(1-\alpha_{i}\right) \alpha_{i+1}+k \gamma\left(1-\alpha_{i+1}\right) \alpha_{i}-$ $k \gamma\left(1-\alpha_{1}\right) \alpha_{i}-k \gamma\left(1-\alpha_{1}\right) \alpha_{i+1}$ (where the first positive terms denote the new information leakage costs associated with these terrorists and the negative terms the old information leakage costs when they were subordinates in the $N-1$ hierarchy). Since the cell is preferred to making $i$ and $i+1$ subordinates to terrorists 1 and 2 , it follows that

$$
\begin{aligned}
& k \gamma\left(1-\alpha_{i}\right) \alpha_{i+1}+k \gamma\left(1-\alpha_{i+1}\right) \alpha_{i}-k \gamma \alpha_{1}\left(1-\alpha_{i}\right)-k \gamma \alpha_{1}\left(1-\alpha_{i+1}\right) \\
< & k \gamma\left(\alpha_{1}+\alpha_{2}\left(1-\alpha_{1}\right) \gamma\right)\left(1-\alpha_{i+1}\right)+k \gamma\left(\alpha_{1}+\alpha_{2}\left(1-\alpha_{1}\right) \gamma\right)\left(1-\alpha_{i}\right)-k \gamma \alpha_{1}\left(1-\alpha_{i}\right)-k \gamma \alpha_{1}\left(1-\alpha_{i+1}\right) \\
= & k \gamma^{2}\left(1-\alpha_{i}\right)\left(1-\alpha_{1}\right) \alpha_{2}+k \gamma^{2}\left(1-\alpha_{i+1}\right)\left(1-\alpha_{1}\right) \alpha_{2} \\
< & k \gamma^{2}\left(1-\alpha_{i}\right) \alpha_{2}+k \gamma^{2}\left(1-\alpha_{i+1}\right) \alpha_{2}
\end{aligned}
$$

The last expression is the information leakage cost associated with the allocation $\widehat{\alpha}$ (that is, the information leakage costs beyond those incurred in anarchy).

Next, we show that the allocation $\widetilde{\alpha}$ has a lower net benefit for the $N-t h \operatorname{link} \widetilde{V}(N)-\widetilde{V}(N-1)$ 
than the allocation $\widehat{\alpha}$, that is, $\widetilde{V}(N)-\widetilde{V}(N-1) \geq \widehat{V}(N)-\widehat{V}(N-1)$. These two incremental values can be written down trivially:

$$
\widetilde{V}(N)-\widetilde{V}(N-1)=t-k \gamma^{2} \sum_{i=3}^{N} \frac{B}{N-1}\left(1-\frac{B}{N-1}\right)=t-k \gamma(N-2) \frac{B}{N-1}\left(1-\frac{B}{N-1}\right)
$$

and

$$
\widehat{c}(N)=t-k \gamma^{2} \sum_{i=3}^{N} \alpha_{2}\left(1-\alpha_{i}\right)=t-k \gamma^{2}(N-2) \alpha_{2}+k \gamma^{2} \alpha_{2} \sum_{i=3}^{N} \alpha_{i}
$$

Since $\sum_{i=3}^{N} \alpha_{i}<B<N-2$, it follows that information leakage costs under $\widehat{\alpha}$ are increasing in $\alpha_{2}$, whose highest value is $\frac{B}{N-1}$ and when it takes this value the information leakage costs are equal to those under $\widetilde{\alpha}$. Thus, $\widetilde{c}(N) \geq \widehat{c}(N) \geq c(N)$. This concludes the proof of Lemma A

Let us now proceed to the proof of Proposition 5.

Suppose now that $t<k \gamma \frac{B}{N}\left(1-\frac{B}{N}\right)$. By Corollary 4 , in this case, the symmetric allocation deters the organization from establishing any link, so this will be the optimal strategy for the enforcement agency. In the rest of the proof we will then assume that $t>k \gamma \frac{B}{N}\left(1-\frac{B}{N}\right)$. In points (1)-(3), we go over all the possible budget allocations and show that the allocation $\widetilde{\alpha}=\left\{0, \frac{B}{N-1}, . ., \frac{B}{N-1}\right\}$ is optimal.

(1) Consider any allocation such that $\alpha_{1}=\alpha_{2}=0$. Then, the organization can reach full efficiency with zero additional information leakage cost with respect to anarchy. To see this, suppose that $\alpha_{1}=\alpha_{2}=0$; then, an organization with the links $\mu_{1 i}=1$ for all $i \in\{2, . ., N\}, \mu_{21}=1$ and $\mu_{i j}=0$ otherwise engenders full trust in the organization. Thus, it must be the case that, in order to prevent links between terrorist and deter efficiency, at most one terrorist can be left with zero probability of detection.

(2) Consider any allocation such that $\alpha_{1}>0$-that is, all the probabilities of detections are set to be positive. Since we are under the assumption that $t>k \gamma \frac{B}{N}\left(1-\frac{B}{N}\right)$, if these probabilities are symmetric, full cooperation will ensue, and the allocation $\widetilde{\alpha}$ cannot do worse than that. Suppose, then, that the allocation is asymmetric - that is, $\alpha_{1}<\frac{B}{N}$. Following the characterization in Proposition 3, the terrorists will then form an optimal organization.

First, suppose the parameters are such that the organization has $N$ links. Then, the allocation we are considering reaches full efficiency, and the allocation $\widetilde{\alpha}$ cannot do worse than that.

Suppose, instead, that the optimal organization given the allocation $\alpha$ we are considering generates $N-1$ links. Then, by the Lemma A, allocation $\widetilde{\alpha}$ performs at least as well.

Finally, suppose that under the allocation $\alpha$ there are $n<N-1$ linked terrorists. We argue that such a structure is impossible. In such organizations, according to Proposition 1, there are three types of terrorists to consider: the top of the hierarchy terrorist 1 , the $N-n-1$ independent terrorists $2, . . N-n$, and the $n$ terrorists who reveal their information to terrorist 1 -that is, $N-n+1, . . N$. Without loss of generality, we will restrict our attention to the allocations that give the same probability of detection to each terrorist in the same category (if the probability is not the same, it is easy to see that it is possible to substitute such probabilities with the average in each category and still obtain the same structure of organization). Let's name such probabilities $\alpha_{1}, \alpha_{2}$ and $\alpha_{N}$ respectively. The probability allocations we are restricting our attention to have to satisfy the 
following constraints:

(i) $0<\alpha_{1} \leq \alpha_{2} \leq \alpha_{N} \leq 1$ (by feasibility and by Proposition 1);

(ii) $k \gamma \alpha_{1}\left(1-\alpha_{2}\right) \geq t$ (it is not optimal for the organization to link the $N-n-1$ independent to terrorist 1$)$;

(iii) $t \geq k \gamma \alpha_{1}\left(1-\alpha_{N}\right)$ (it is optimal for the organization to link the $n$ terrorists to terrorist 1 );

(iv) $\alpha_{1}+(N-n-1) \alpha_{2}+n \alpha_{N} \leq B$ (the resource constraint).

Note that $k \gamma \alpha_{1}\left(1-\alpha_{2}\right) \leq k \gamma \alpha_{2}\left(1-\alpha_{2}\right) \leq k \gamma \frac{B}{N}\left(1-\frac{B}{N}\right)$ since $\alpha_{2} \leq \frac{B}{N}<\frac{1}{2}$ (otherwise either the (iv) or is violated or it cannot be that $\alpha_{1} \leq \alpha_{2} \leq \alpha_{N}$ ), but then (ii) cannot hold since $t>k \gamma \frac{B}{N}\left(1-\frac{B}{N}\right)$. If follows that such a structure is impossible.

(3) In points (1)-(2) we showed that if $t>k \gamma \frac{B}{N}\left(1-\frac{B}{N}\right)$, all the allocations such that $\alpha_{1}=\alpha_{2}=0$ or $\alpha_{1}>0$ are (weakly) dominated by allocation $\widetilde{\alpha}$. Finally, let us consider an allocation such that $\alpha_{1}=0$ and $\alpha_{2}>0$. Under this allocation, it is clear that an organization with $N-1$ linked terrorists can arise costlessly. Thus, the best the enforcement agency can do is to try to prevent the $N-t h$ link from arising. Observe that, if $\alpha_{1}=0$, the characterization in Proposition 2 yields, for each $i \in\{4, . ., N\}, \rho(i-1 . i) \leq 1-\alpha_{2}$ (easy to check since $\alpha_{2} \leq \alpha_{j}$ for all $j \in\{3, . ., N\}$ ). Then, in the optimal organization, all the terrorists are linked to terrorist 1, without binary cells (besides the cell $\{1,2\})$. Then, the cost of the $N-t h$ link for the organization is $k \gamma \alpha_{2} \sum_{i=3}^{N}\left(1-\alpha_{i}\right)$, and it is maximized (under the constraints $\alpha_{2} \leq \alpha_{i}$ for all $i$ and $\sum_{i=2}^{N} \alpha_{1}=B$ ) by $\alpha_{i}=\frac{B}{N-1}$ for all $i \in\{2, . ., N\}$, which is allocation $\widetilde{\alpha}$ 